\title{
Application of Health Technology Assessment for Oncology Care in India: Implications for Ayushman Bharat Pradhan Mantri Jan Aarogya Yojana
}

\author{
Nidhi Gupta10 \\ Shankar Prinja ${ }^{2}$ \\ ${ }^{1}$ Department of Radiation Oncology, Government Medical College \\ and Hospital, Chandigarh, India \\ 2 Department of Community Medicine and School of Public Health, \\ Post Graduate Institute of Medical Education and Research, \\ Chandigarh, India \\ ${ }^{3}$ Department of Health Research, Ministry of Health and Family \\ Welfare, Government of India, New Delhi \\ ${ }^{4}$ Department of Women and Child Development, Government of \\ India, New Delhi \\ ${ }^{5}$ National Health Authority, Ministry of Health and Family Welfare, \\ Government of India, New Delhi
}

Rakesh Gupta ${ }^{4}$ Anu Nagar ${ }^{3}$ Praveen Gedam 5

Ind J Med Paediatr Oncol 2021;42:479-483.

\author{
Address for correspondence Shankar Prinja, MD, Professor of Health \\ Economics Department of Community Medicine and School of Public \\ Health, Postgraduate Institute of Medical Education and Research \\ (PGIMER), Chandigarh 160012, India \\ (e-mail: shankarprinja@gmail.com).
}

\begin{abstract}
Keywords

- cost-effectiveness

- health economics

- health insurance

- Health Technology Assessment

- medical oncology

- priority setting

- universal health coverage

A health system is considered efficient when it provides maximum health gains to the population from the available resources. Newer drugs, diagnostics and treatment strategies aim to improve the health of the population, however, they come at an increased cost. Therefore, for an efficient health system, it needs to be decided if the extra cost being incurred is justified to achieve the extra health gains. In this regard, health technology assessment (HTA) helps to make evidence informed decisions by evaluating relative cost and benefits of the available interventions. Economic evidence generated by HTA can also be used in framing standard treatment guidelines (STGs) for high-cost cancer care. In multi-payer systems like India, the decisions regarding the clinical management of patients are taken based on the patients' ability to pay, which creates inequities in utilization of healthcare. Ayushman Bharat Pradhan Mantri Jan Aarogya Yojana (AB PM-JAY) offers an opportunity to ensure equity as it reduces financial barriers, besides having a potential to affect efficiency by including only costeffective interventions in the benefit package. As a result, informed clinical decisions based upon HTA evidence can make cancer treatment more efficient, equitable and affordable for the patients.
\end{abstract}

DOI https://doi.org/ 10.1055/s-0041-1740536. ISSN 0971-5851. (c) 2021. Indian Society of Medical and Paediatric Oncology. All rights reserved.

This is an open access article published by Thieme under the terms of the Creative Commons Attribution-NonDerivative-NonCommercial-License, permitting copying and reproduction so long as the original work is given appropriate credit. Contents may not be used for commercial purposes, or adapted, remixed, transformed or built upon. (https://creativecommons.org/ licenses/by-nc-nd/4.0/)

Thieme Medical and Scientific Publishers Pvt. Ltd., A-12, 2nd Floor, Sector 2, Noida-201301 UP, India 


\section{Introduction}

The World Health Organization, in its report in 2000, promulgated achieving the best-possible level of societal wellbeing as the overarching goal for all the healthcare systems present worldwide. ${ }^{1}$ But, there are several intermediate end points or values that should be achieved first. These intermediate goals include the ability to provide necessary and good quality healthcare services, delivered in a dignified way, at a reasonable cost that does not impose any financial hardship to the patient. To maximize the health benefits to the population with the available resources, the health system needs to be efficient and should ensure that the services are delivered in an equitable fashion to the population.

From the financial standpoint, improving efficiency or the value of money being spent is one of the important ways to enhance the level of population health. There are four key stakeholders involved in the efficient delivery of the health services: the policy makers, purchasers of healthcare services, healthcare providers or clinicians, and finally consumers or patients. Each one of them has a different perception and aspiration for healthcare efficiency. The policy makers ensure that they get the highest value for the money being spent, besides the programs being delivered equitably and that enhance the level of financial risk protection. The purchaser designs health benefit packages (HBPs) of universal coverage schemes, and engages with public and private providers in strategic purchasing. It aims to set prices of the services to be delivered that reflect its value and set the right provider incentives. From the point of view of providers or clinicians, they need to adhere to certain standard treatment guidelines that enhance the level of technical efficiency. For consumers, the value means the overall level of quality of care and satisfaction they achieve with the delivery of healthcare services.

Health Technology Assessment (HTA) aims to evaluate the relative costs and benefits of any new drug, device, and treatment or delivery strategy.

These were considered in terms of their clinical effectiveness, cost-effectiveness, and issues related to ethics, acceptability, and equity. ${ }^{2}$ One of the key outcomes of an HTA study is estimating the incremental cost per unit gain in health benefits with application of the new intervention. ${ }^{3,4}$ This needs to be weighed against a value-judgment of whether the extra cost being incurred to produce an extra health benefit is worth spending.

In this paper, we focus on the role of HTA for determining treatment choices from a clinician's perspective. In particular, we focus on the field of oncology that involves decisions for new costly drugs, diagnostics, and treatment strategies. In the next section, we describe how HTA and economic evidence can be used to influence decisions with respect to standard treatment guidelines. We use examples of recent HTA studies to illustrate this point. Next, we describe the health financing situation of India, to highlight the difficulty of integrating HTA evidence for standard treatment guidelines, clinical decision-making, and reimbursement deci- sions. We finally conclude by describing the future prospects on how informed clinical decisions based upon HTA evidence can make cancer treatment more efficient and affordable to the patient.

\section{Clinical Context}

Oncology is the only specialty within the medical field that has had the most number of progressive scientific developments. This leads to generation of new evidences for better ways of practice every day. ${ }^{5}$ The new evidence aims at providing better disease-free survival (DFS) and overall survival (OS) rates when compared with the available standard of care. Various international and national guidelines continue to be updated as per the evidence from current state of the art clinical trials, so that all patients can be offered preventive strategies, diagnostic workup, treatment, and supportive care. This will hopefully lead to best-possible health outcomes. ${ }^{6-8}$ These guidelines for the management of oncology patients give evidence-based recommendations based on the disease stage, histology, investigative workup, clinical parameters, performance status, and adverse effects. The guidelines are classified into various categories based on the level of evidence. For example, category 1 is described in the most commonly used. National Comprehensive Cancer Network (NCCN) guidelines refer to recommendations generated based upon high level of evidence with uniform NCCN consensus that the intervention is appropriate. ${ }^{6}$ The preferred interventions are usually based on superior efficacy and safety. But, the factors such as cost and cost-effectiveness are not considered here.

Recently, however, the guidelines being prepared by the Indian National Cancer Grid do attempt to stratify the advice based on resource availability in terms of essential resources, desired resources, and what could be done if there is no resource constraint. ${ }^{8}$ However, these guidelines also do not explicitly use evidences proving cost-effectiveness. Further, all these guidelines provide multiple preferred options for the same set of clinical situations, and just one preferred option is not separately considered. The choice from among these clinical options is based on the informed decisions taken by the patients. Next, we use examples from three economic evaluations to illustrate how the guidelines could be informed using such evidence.

Breast cancer is the most common cancer among females in India. ${ }^{9}$ The adjuvant trastuzumab is an essential component in the treatment of human epidermal growth factor receptor 2 (HER2) positive breast cancer cases. All international guidelines continue to recommend 1 year of adjuvant trastuzumab for HER2 positive breast cancer patients along with chemotherapy. ${ }^{10,11}$ However, a shorter dose duration of the drug for 9 weeks or 6 months has also shown to be efficacious in this scenario. In a meta-analysis performed in India, the 5-year DFS rate with 9 weeks of trastuzumab was reported to be $85.42 \%$ versus $87.12 \%$ with 1 year of drug use. $^{12}$ Similarly, the OS rate was $92.39 \%$ and $93.46 \%$ with 9 weeks and 1 year of trastuzumab, respectively. While guidelines continue to recommend 1 year of trastuzumab, 
only $8 \%$ of the patients who are candidates for trastuzumab receive it due to its cost. ${ }^{13}$ Among this set of patients who use trastuzumab, $50 \%$ of them are enrolled in some form of clinical trials and receive the drug for free. So practically, only $40 \%$ of the eligible patients are able to afford this expensive molecule for treatment. ${ }^{14}$ This clearly shows that cost constraints determine access to treatment, and those who need treatment are not able to receive care, which is likely to lower the health benefits. This clearly indicates inefficient allocation of resources.

In a recent economic evaluation, 1 year of trastuzumab use was reported to result in a gain of 1.29 quality-adjusted life years (QALYs) at an additional cost of 178,877 (US\$2,558) per QALY gained. ${ }^{15}$ Overall, there was only $4 \%$ probability for 1 year of trastuzumab to be cost-effective. The study concluded that 9 weeks and 6 months of therapy is cost-effective in India. Though 1 year of trastuzumab gives greater health benefits, the incremental cost incurred with 1 year of trastuzumab is not justified given the threshold for cost-effectiveness in India. However, if the cost of trastuzumab is brought down to one-third of its current cost by means of price regulation, then there is $80 \%$ probability that 1 year of trastuzumab becomes cost-effective in India. The guidance for use of trastuzumab should accordingly be revised in clinical guidelines for India.

Another study was performed to estimate the cost-effectiveness of sorafenib versus best supportive care in patients with advanced unresectable hepatocellular carcinoma with extrahepatic spread, vascular invasion, or progression after local therapies. It was found out that sorafenib incurs $507,520(\$ 7,861)$ per QALY gained. ${ }^{16}$ The study concluded that sorafenib is not cost-effective for use in advanced hepatocellular carcinoma treatment in India. In a third recent study, use of the adjuvant temozolomide along with radiation therapy for treatment of glioblastoma multiforme was not found to be cost-effective. ${ }^{17}$

We see from the above examples that clinical guidelines based on effectiveness data may not be in-sync with economic evidence. Clinical guidelines continue to recommend dosage of 1 year of trastuzumab, sorafenib, as well as temozolomide. ${ }^{15-17}$ Integration of this with economic evidence specific to India in the clinical recommendations will help to attain greater health benefits at a population level. This will only be possible by optimal utilization of the limited resources. Each of these economic evaluations also indicates reasonable prices for these drugs. This is possible only if these prices are reduced through some regulation or bulk purchasing.

\section{Financing Context: Unearthing the Real Issue}

The National Health Service (NHS) in the United Kingdom has a single payer system and is the largest purchaser of healthcare. It pays for the health care of the entire population. ${ }^{18}$ It also provides final management guidelines, based on costeffectiveness evidence that is generated by the National Institute for Health and Care Excellence. ${ }^{19}$ NHS can also exert its monopsonistic power to negotiate prices with the pharmaceutical companies, as has been evident in the case of trastuzumab. 20

India, on the other hand, has a multipayer system. While $14 \%$ of the rural population and $19 \%$ of the urban population has some form of health insurance coverage, the majority (93\% of rural and $47 \%$ of urban population) of this insured population is covered under the tax-funded Ayushman Bharat Pradhan Mantri Jan Aarogya Yojana (AB PM-JAY) and other State-funded health insurance schemes. ${ }^{21}$ Most of these schemes include treatment of cancer in the HBP too. However, nearly $72 \%$ of the total health expenditure in India is incurred by households. ${ }^{22}$

In such a situation, the decisions about the clinical management of the patients are taken based on a mutual discussion between the clinician and the patient. ${ }^{22}$ Here, the clinician discusses the various available treatment options with the patient in terms of the risk-benefit ratio. Benefits are described in terms of the rates of OS and DFS. Risks that can be incurred in terms of the adverse effects associated with these treatment options are also explained. Then, the clinician discusses with the patient the cost incurred with each of these treatment options. Based upon the ability of the patient to pay, the choice of treatment is made. Since the government payer or AB PM-JAY does not yield large market share, it is limited in terms of its power to influence clinical decisions or adherence to standard treatment guidelines.

Thus, we see that a strong driver for clinical decisionmaking in this multipayer system is the patient's ability to pay. This fallout of our health financing situation, where access to care is contingent upon ability to pay, leads to reduced access and unmet needs for those who require treatment. As a result, it is inefficient. Moreover, the poor patients are the hardest hit. ${ }^{23-25}$ This creates inequities in utilization and supply of health care.

\section{Solution}

To ensure the application of HTA for value-based healthcare, India would need to move toward a single payer or a single purchaser system, which will purchase healthcare on behalf of the entire population. Hence, it will be able to exercise some degree of control and regulation in India. Currently, the predominant option is the AB PM-JAY. There are three ways in which the $A B$ PM-JAY can influence uptake of HTA evidence in clinical decision-making. First, the HBPs should be determined based on the HTA evidence. Only those treatments that are found to be cost-effective should be included in the standard treatment workflow for reimbursement. Second, the provider payment rates that are set for each HBP should be based on the cost of services that are found to be value-based. Finally, when the providers are reimbursed, there should be incentives (such as higher rating of these empaneled hospitals, or higher price reward) for providers to adhere to these standard treatment guidelines, which should be driven by evidence. This would have twin benefits. First, it would lead to a culture of evidence-based practice that would be efficient. Second, it would reduce the 
incidents regarding the practice of "balance-billing" to patients. ${ }^{26}$ In the absence of such regulation or incentive for adherence to standard guidelines, the clinicians may have a perverse incentive to provide unnecessary care, and charge the same to patients, over and above the claim submitted to AB PM-JAY.

Realization of this utopian, but realistic situation would need to be supplemented with appropriate nudging through a policy environment that supports the uptake of HTA evidence. The global experience suggests that the uptake of HTA evidence is favored by appropriate legislative mandate. Several European countries make the decisions for reimbursement legally binding on HTA evidence. Closer home, several South Asian countries such as Philippines, Indonesia, Malaysia, Singapore, and Vietnam have either a legislative mandate or other legal provisions or by-laws that mandate the use of HTA for a certain category of drugs, thereby providing more teeth to their respective country HTA bodies.

India also created its HTA body in 2018-The Health Technology Assessment in India (HTAIn)-the Secretariat for which is located in the Department of Health Research. ${ }^{27,28}$ Several HTA studies, commissioned by the HTAIn, have led to uptake in policy decisions. ${ }^{29-31}$ A study that evaluates the value-based prices for several anticancer drugs in India is currently in progress. There needs to be institutional mechanisms for a greater interaction between the HTAIn and the AB PM-JAY to enable uptake of HTA evidence. To begin with, any new package added or new drug or treatment to be reimbursed should be subjected to HTA.

A draft legislation-“The HTA Bill"-has been created, which is being considered to be tabled in the Indian Parliament. In its current form, the HTA Bill offers recommendatory status, without any statutory powers. A way forward would be to position the linkage between cost-effectiveness threshold (CET) and the right to health. First, the CET in India should be determined, and then it should serve as a binding rule for the Government to provide proven and cost-effective services. $^{3}$ Second, the Central Government's allocations to the State Government, as a part of schemes such as National Health Mission, could be made contingent on the State adhering to this condition of providing proven cost-effective services. So, this has a broader aspirational objective to use HTA to nudge the Government toward universal health coverage and providing the right to health to the patients.

The second legislative issue concerns the pricing of pharmaceuticals. The Government has two ways of regulating the drug prices: inclusion of the drugs in the essential drug list (EDL) or regulating the trade margin. If a drug is included in the EDL, then the Government can regulate its price (Government proposes a price control order formula). An alternative viewpoint could be an application of HTA to determine a "value-based price." Third, as part of the State-level medical service corporations, the use of HTA should become mandatory for procurement of drugs and devices under these medical service corporations (to identify what should be procured, and at what price).
There are several other criteria and processes that can be used for priority setting. ${ }^{32,33}$ These methods include Multicriteria Decision Analysis, Program Budgeting and Marginal Analysis. However, considering that the systems for HTA have been institutionalized in India, and its capacity is being developed, it is more pragmatic to further build upon the work that has been already done.

\section{Conclusion}

Overall, the application of HTA has benefits of making health services, especially related to oncology practice, efficient, affordable, and equitable. The problem lies with the Indian health financing situation, which leads to multipayer system where decision to use health service is contingent upon the payment capabilities of the patient. Investing adequately in health to create a single large purchaser would be the first step. ${ }^{34,35}$ Second, decisions of the AB PM-JAY would need to be linked with HTA evidence. Conducive legislative measures to promote HTA would go a long way in realization of the aspirations of universal coverage and efficient health system for Indian patients.

\section{Sources of Support}

No support in the form of grants, equipment, or drugs was obtained to prepare this manuscript.

\section{Author Declaration}

The manuscript has been read and approved by all the authors. All the authors meet the requirements for authorship, and each author believes that the manuscript represents honest work.

Conflict of Interest

None of the authors declared any conflict of interest.

\section{References}

1 World Health Organization The World Health Report 2000. Health Systems: Improving Performance. Geneva, 2000. https://apps. who.int/iris/handle/10665/42281. Accessed December 1, 2021

2 World Health Organization. WHO | Health technology assessment. [online]. https://www.who.int/medical_devices/assessment/en/ Accessed Accessed December 1, 2021.

3 Prinja S, Sundararaman T, Muraleedharan VR. Cost-effectiveness threshold and health opportunity cost. Econ Polit Wkly 2020;55 (02):19

4 Chi YL, Blecher M, Chalkidou K, et al. What next after GDP-based cost-effectiveness thresholds? Gates Open Res 2020;4:176. Doi: 10.12688/gatesopenres.13201.1 eCollection 2020

5 World Health Organization. WHO List of Priority Medical Devices for Cancer Management. Geneva: World Health Organization; 2017

6 National Comprehensive Cancer Network. 2021NCCN Guidelines: Treatment by Cancer Type. [online]. <https://www.nccn.org/ guidelines/category_1> Accessed December 1, 2021

7 ICMR Consensus Documents for Cancer Management. India Against Cancer. http://cancerindia.org.in/icmr-consensus-documents-for-cancer-management/ Accessed December 1, 2021

8 National Cancer Grid Draft Guidelines. 2020. Draft Guidelines 2020 - NCG. https://tmc.gov.in/ncg/index.php/guidelines/draftguidelines-2020 Accessed December 1, 2021 
9 Sung H, Ferlay J, Siegel RL, et al. Global cancer statistics 2020: GLOBOCAN estimates of incidence and mortality worldwide for 36 cancers in 185 countries. CA Cancer J Clin 2021;71(03): 209-249 Epub ahead of print 10.3322/caac.21660

10 Guidelines Detail- Breast Cancer. National Comprehensive Cancer Network. https://www.nccn.org/guidelines/guidelines-detail? category=1\&amp;id=1419 Accessed December 1, 2021

11 Consensus Document for Management of Breast Cancer. Indian Council of Medical Research. [online]. https://main.icmr.nic. in/sites/default/files/guidelines/Breast_Cancer.pdf Accessed December 1,2021

12 Gulia S, Kannan S, Badwe R, Gupta S. Evaluation of 1-year vs shorter durations of adjuvant trastuzumab among patients with early breast cancer: an individual participant data and trial-level meta-analysis. JAMA Netw Open 2020;3(08):e2011777. Doi: 10.1001/jamanetworkopen.2020.11777

13 Ghosh J, Gupta S, Desai S, et al. Estrogen, progesterone and HER2 receptor expression in breast tumors of patients, and their usage of HER2-targeted therapy, in a tertiary care centre in India. Indian J Cancer 2011;48(04):391-396

14 Vijaykumar DK, Arun S, Abraham AG, Hopman W, Robinson AG, Booth CM. Breast cancer care in South India: is practice concordant with national guidelines? J Glob Oncol 2019;5:1-7

15 Gupta N, Verma RK, Gupta S, Prinja S. Cost effectiveness of trastuzumab for management of breast cancer in India. JCO Glob Oncol 2020;6:205-216

16 Gupta N, Verma RK, Prinja S, Dhiman RK. Cost-effectiveness of sorafenib for treatment of advanced hepatocellular carcinoma in India. J Clin Exp Hepatol 2019;9(04):468-475

17 Gupta N, Prinja S, Patil V, Bahuguna P. Cost effectiveness of temozolamide for treatment of glioblastoma multiforme in India. J Global Oncol 2021;7:108-117 Jan 15: DOI https://doi.org/ $10.1200 / G 0.20 .00288$

18 Oliver A. The single-payer option: a reconsideration. J Health Polit Policy Law 2009;34(04):509-530

19 Owen L, Pennington B, Fischer A, Jeong K. The cost-effectiveness of public health interventions examined by NICE from 2011 to 2016. J Public Health (Oxf) 2018;40(03):557-566

20 NHS England. NHS England strikes deal to make innovative breast cancer drug routinely available on the NHS [Internet]. England.nhs. uk. 2021 [cited 18 July 2021]. https://www.england.nhs.uk/2017/ 06/breast-cancer-drug-routinely-available-on-the-nhs/. Accessed December 1, 2021

21 National Sample Survey Office. Health in India- NSS 75th Round [Internet]. New Delhi: National Sample Survey Office, Ministry of Statistics and Programme Implementation; July 2020. http://mospi. nic.in/sites/default/files/publication_reports/NSS\%20Report\%20no. \%20586\%20Health \%20in\%20India.pdf. Accessed December 1, 2021

22 National Health Accounts Technical Secretariat. National Health Accounts Estimates for India 2016-17 [Internet]. New Delhi: National Health Systems Resource Centre; 2020. http://nhsrcindia. org/sites/default/files/FINAL\%20National\%20Health\%20Accounts\%
202016- 17\%20Nov\%202019-for\%20Web.pdf. Accessed December 1, 2021

23 Prinja S, Kumar MI, Pinto AD, Jan S, Kumar R. Equity in hospital services utilisation in India. Econ Polit Wkly 2013;xlviil(12): 52-58

24 Prinja S, Aggarwal AK, Kumar R, Kanavos P. User charges in health care: evidence of effect on service utilization \& equity from north India. Indian J Med Res 2012;136(05):868-876

25 Prinja S, Kanavos P, Kumar R. Health care inequities in north India: role of public sector in universalizing health care. Indian J Med Res 2012;136(03):421-431

26 Prinja S, Chauhan AS, Karan A, Kaur G, Kumar R. Impact of publicly financed health insurance schemes on healthcare utilization and financial risk protection in India: a systematic review. PLoS One 2017;12(02):e0170996

27 Prinja S, Downey LE, Gauba VK, Swaminathan S. Health technology assessment for policy making in India: current scenario and way forward. Pharmacoecon Open 2018;2(01):1-3

28 Downey LE, Mehndiratta A, Grover A, et al. Institutionalising health technology assessment: establishing the Medical Technology Assessment Board in India. BMJ Glob Health 2017;2(02): e000259. Doi: 10.1136/bmjgh-2016-000259

29 Bahuguna P, Prinja S, Lahariya C, et al. Cost-effectiveness of therapeutic use of safety-engineered syringes in healthcare facilities in India. Appl Health Econ Health Policy 2020;18(03): 393-411. Doi: 10.1007/s40258-019- 00536-w

30 Chugh Y, Dhiman RK, Premkumar M, Prinja S, Singh Grover G, Bahuguna P. Real-world cost-effectiveness of pan-genotypic Sofosbuvir-Velpatasvir combination versus genotype dependent directly acting anti-viral drugs for treatment of hepatitis $C$ patients in the universal coverage scheme of Punjab state in India. PLoS One 2019;14(08):e0221769

31 Prinja S, Rajsekhar K, Gauba VK. Health technology assessment in India: reflection \& future roadmap. Indian J Med Res 2020;152 (05):444-447

32 Kaur G, Prinja S, Lakshmi PVM, Downey L, Sharma D, Teerawattananon Y. Criteria used for priority-setting for public health resource allocation in low- and middle-income countries: a systematic review. Int J Technol Assess Health Care 2019;35 (06):474-483

33 Thokala P, Devlin N, Marsh K, et al. Multiple criteria decision analysis for health care decision making-an introduction: report 1 of the ISPOR MCDA Emerging Good Practices Task Force. Value Health 2016;19(01):1-13

34 Prinja S, Bahuguna P, Pinto AD, Sharma A, Bharaj G, Dhiman V, Tripathy JP, Kaur M, Kumar R. Cost of Universal Health Care Provision in India: a Model-Based Analysis. PLoS One 2012;7 (01):e30362

35 Singh D, Prinja S, Bahuguna P, et al. Cost of scaling-up comprehensive primary health care in India: Implications for universal health coverage. Health Policy Plan 2021;36(04): 407-417 